\title{
Regional to Global Evolution of Impacts of Parameterized Mountain-Wave Drag in the Lower Stratosphere
}

\author{
CHRISTOPHER G. KRUSE \\ Advanced Study Program, National Center for Atmospheric Research, Boulder, Colorado
}

(Manuscript received 28 January 2019, in final form 2 January 2020)

\begin{abstract}
Mountain ranges are regional features on Earth, as are the regions of mountain-wave drag (MWD) exerted by dissipating atmospheric gravity waves generated by flow over them. However, these regional drags have significant global- or zonal-mean impacts on Earth's atmospheric general circulation (e.g., slowing of the polar night jet). The objective of this work is to understand the regional to global evolution of these impacts. The approach is to track the evolution of MWD-generated potential vorticity (PV) over the winter using the Whole Atmosphere Community Climate Model (WACCM). Within an ensemble of winter-long runs with and without MWD, lower-stratospheric PV is generated over mountains and advected downstream, generating large-scale PV banners. These PV banners are diffused but survive this diffusion and are reinforced over downstream mountain ranges, accumulating into zonal-mean or global features within WACCM. A simple 2D model representing sources, advection, and diffusion of "passive PV" recreates the salient features in the WACCM results, suggesting the winter-long evolution of MWD-generated PV can be crudely understood in terms of horizontal advection and diffusion within a global vortex. In the Northern Hemisphere, cyclonic, equatorward PV banners accumulate zonally into a single zonally symmetric positive PV anomaly. The anticyclonic, poleward PV banners also accumulate into a zonally symmetric feature, but then diffuse over the North Pole into a negative PV polar cap. In the Southern Hemisphere, the same processes are at work, though the different geographic configuration of mountain ranges leads to different patterns of impacts.
\end{abstract}

\section{Introduction}

Mountain waves (MWs) are atmospheric internal gravity waves generated by stratified flow over mountains. When MWs are generated by a mountain, a positive (negative) pressure perturbation develops upstream (downstream) of the mountain, exerting a net pressure drag on the mountain and transferring atmospheric momentum to the Earth. An equal and opposite force is exerted by the mountain on the atmosphere, which is propagated upward by the generated MW and imparted wherever the MW is attenuated (Teixeira 2014). Because mountains and the MWs they generate are partly (largely) unresolved in weather (climate) models, they are conventionally parameterized.

The drag force deposited by mountain-wave drag (MWD) parameterizations is important in both weather and climate. The inclusion of these parameterizations has led to improvements in forecast skill (e.g., Miller et al. 1989; Sandu et al. 2019) and in the wintertime

Corresponding author: Christopher G. Kruse, ckruse@ucar.edu zonal-mean zonal winds in the upper troposphere and lower stratosphere (e.g., Palmer et al. 1986; McFarlane 1987). MWs influence climate in the stratosphere both directly, via the drag they deposit, and indirectly by altering the mean fields upon which large-scale waves propagate, which can alter regions of large-scale wave momentum deposition (e.g., Sigmond and Scinocca 2010). Figure 1 summarizes the impact of an MWD parameterization on Northern and Southern Hemisphere wintertime zonal-mean zonal winds in 28 -yr freerunning Whole Atmosphere Community Climate Model (WACCM) simulations with and without parameterized MWD. In both hemispheres, the largest impacts are seen at $z=40-50 \mathrm{~km}$ as decelerations of the stratospheric polar night jet, though significant decelerations do extend downward into the upper troposphere and lower stratosphere. Larger impacts occur in the Northern Hemisphere due to the significantly larger area occupied by mountains.

Clearly, MWs have a significant global- or zonal-mean impact on the general circulation. However, mountainous areas and, hence, areas experiencing MWD are 


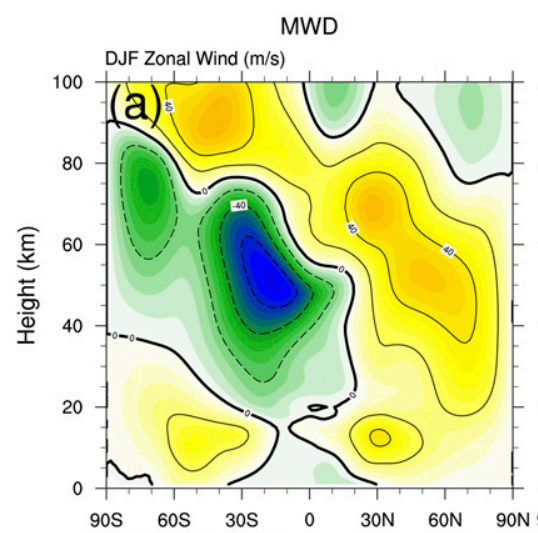

\section{DJF Zonal Wind $(\mathrm{m} / \mathrm{s})$}

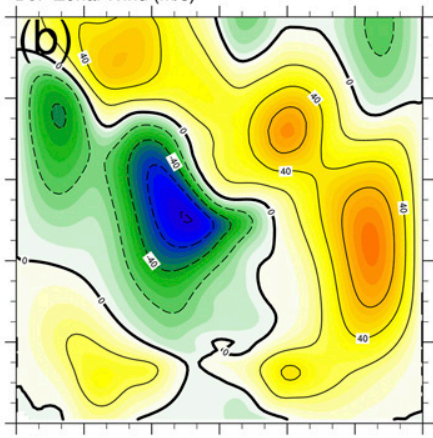

JJA Zonal Wind $(\mathrm{m} / \mathrm{s})$



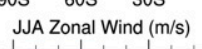

JJA Zonal

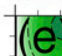

FIG. 1. Wintertime-, zonal-mean zonal winds $\left(\mathrm{m} \mathrm{s}^{-1}\right)$ from 28-yr-long free-running WACCM simulations (a),(d) with and (b),(e) without parameterized MWD. (c),(f) The MWD - NoMWD differences are shown. Results from Northern (Southern) Hemisphere winter are shown in the top (bottom) row. WACCM was configured to represent present-day climate.

regional (Fig. 2). The objective here is to understand how impacts of regional forcings by MWD become global and affect the zonal mean. The approach is to track potential vorticity (PV) generation by the MWD parameterization, its advection, and its long-term evolution over a winter in a general circulation model.

At low levels, $\mathrm{PV}$ is generated in flow over mountains either by frictional contact of split flow with the flanks of a mountain or through momentum deposition by turbulent MW breakdown (Smith 1989). Aloft, PV is generated through the latter process, which tends to deposit momentum inhomogeneously in space (e.g., Fig. 11 of Kruse et al. 2016). In both cases, the generated $\mathrm{PV}$ is conservatively advected downstream, creating PV banners (Schär et al. 2003; Chen et al. 2007; Kruse et al. 2016; Menchaca and Durran 2018). Lateral instabilities
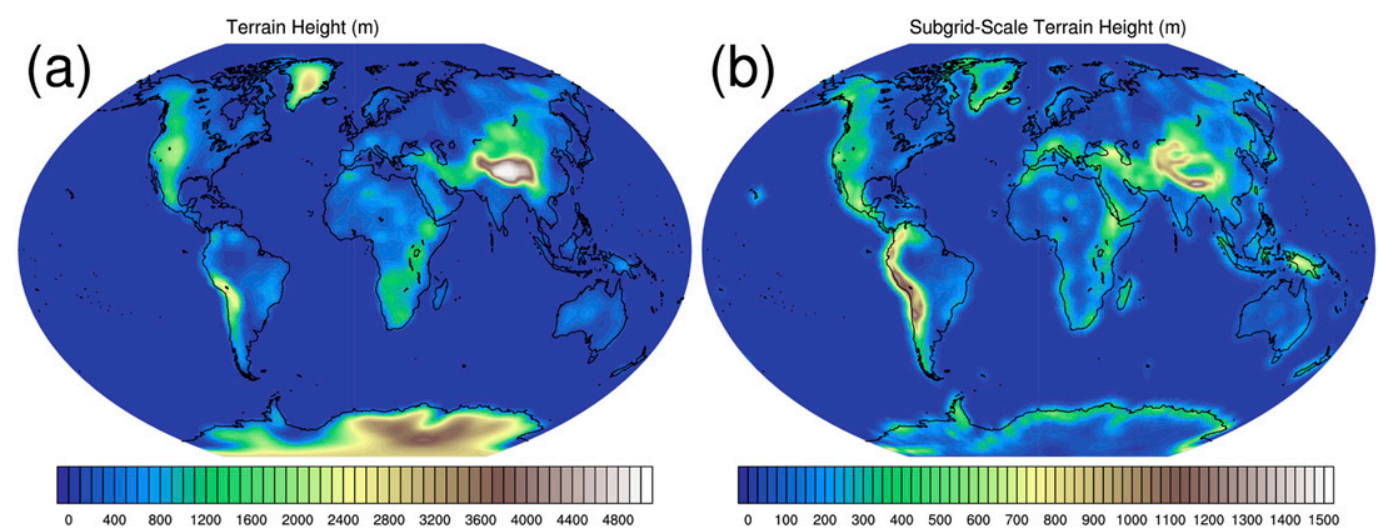

FIG. 2. (a) Resolved and (b) unresolved terrain heights (m) in the WACCM GCM. Note the different color scales. 
tend to homogenize MW-scale PV banners, resulting in larger-scale positive (negative) PV anomalies to the left (right) of the regional-mean drag vector (Chen et al. 2007; Menchaca and Durran 2018). Chen et al. (2007) showed that downstream wind differences in mesoscale simulations with and without a mountain are largely reproduced by the flow field from inverting the MWDgenerated PV. Hence, if it is possible to understand the evolution of MWD-generated PV, a portion of the final flow field can be attributed to MWD. Here, the evolution of PV generated by MWD parameterized in WACCM is tracked to study the regional to global evolution of MWD impacts.

The paper is organized as follows: In section 2, the WACCM and experimental setup are presented. In section 3, the short-term and winter-long evolution of MWD-generated PV is presented and discussed. Section 4 includes a description and results of a $2 \mathrm{D}$ horizontal diffusion model used to better interpret the WACCM results. A summary and discussion are provided in the final section.

\section{GCM configuration and experimental design}

\section{a. GCM configuration}

To study the regional to global evolution of impacts of the MWD parameterization, the Community Earth System Model (CESM), version 1.2.2.1, was used. The deep $(z=0 \approx 150 \mathrm{~km})$ WACCM configuration of CESM was selected to represent year 2000 climate (component set "F_2000_WACCM5"). NCAR's Community Atmosphere Model, version 5, is the atmospheric general circulation model (GCM) within this configuration. Sea ice and sea surface temperatures were prescribed based on present-day climatology. A $1.9^{\circ}$ by $2.5^{\circ}$ resolution latitude-longitude grid was used. The CESM configuration described above is hereafter referred to as "WACCM."

The MWD parameterization used in this version of WACCM is largely based on that described by McFarlane (1987). Subgrid-scale terrain is used to compute a source MW amplitude and is simply characterized by twice its standard deviation within a grid cell (Fig. 2). Two-dimensional, linear, hydrostatic, steady, WKB theory is used to compute MW amplitudes with height given this source amplitude and a vertically varying environment assumed to be horizontally homogenous. Within this theory, the vertical fluxes of horizontal momentum (hereafter, momentum fluxes) by nonattenuating MWs are constant with height, resulting in wave-amplitude growth with decreasing density. A saturation assumption (Lindzen 1981) is used to constrain
MW amplitudes so that they cannot induce static instability, which reduces momentum fluxes and results in MWD. The source drag characterization is isotropic; the source momentum flux vector is parallel to and opposes the source wind vector. If the subgrid-scale terrain is high enough that this linear theory predicts a supersaturated wave, the source amplitude is limited to the saturation value. This initialized MW is then allowed to grow with altitude, eventually saturate, and deposit momentum aloft. For further details, see Neale et al. (2010).

The primary experiment discussed here was conducted by running WACCM without MWD (labeled NoMWD) for 20 years starting 1 December 1999. Then, at the start of every Northern and Southern Hemisphere winter (1 December and 1 June, respectively), a WACCM run with MWD (labeled MWD) was branched off the NoMWD run and integrated for three months. The two runs with and without MWD over a particular winter are together referred to as a single branch. The MWD and NoMWD runs are compared to investigate the evolution of the influences of MWD.

\section{b. Definitions}

Differenced fields (e.g., for variable $a$ ) are defined by

$\Delta a_{i}(x, y, z, t)=a_{i_{\mathrm{MWD}}}(x, y, z, t)-a_{i_{\mathrm{NoMWD}}}(x, y, z, t)$,

where subscript $i$ indicates branch or year number. Differences within a single branch (e.g., $\Delta a_{1}$ ) show regional impacts of introducing MWD, at least within the first $\approx 5-10$ days of the branch time. After about 10 days, the two solutions diverge, and differences for a single branch become partly due to the addition of parameterized MWD and partly due to different resolved variability (e.g., Rossby waves, see next section) in the NoMWD and MWD runs. Averaging over all 20 branches (i.e., winters), that is

$$
\widetilde{\Delta a}(x, y, z, t)=\frac{1}{N_{b}} \sum_{i=1}^{N_{b}} \Delta a_{i}(x, y, z, t),
$$

averages out random differences due to different resolved variability. Systematic differences that remain are direct (e.g., decelerations by MWD) and indirect (e.g., changes in decelerations by resolved waves due to altered propagation trajectories) impacts of the MWD parameterization. Branch-averaged differences can also be interpreted as differences in ensemble means, which is how such averages will be referred to hereafter.

The variable of interest here is PV, defined by

$$
\mathrm{PV}=\frac{\boldsymbol{\omega}_{a} \cdot \nabla \chi}{\rho}=\operatorname{EPV}\left(\frac{\theta}{\theta_{0}}\right)^{-\alpha},
$$




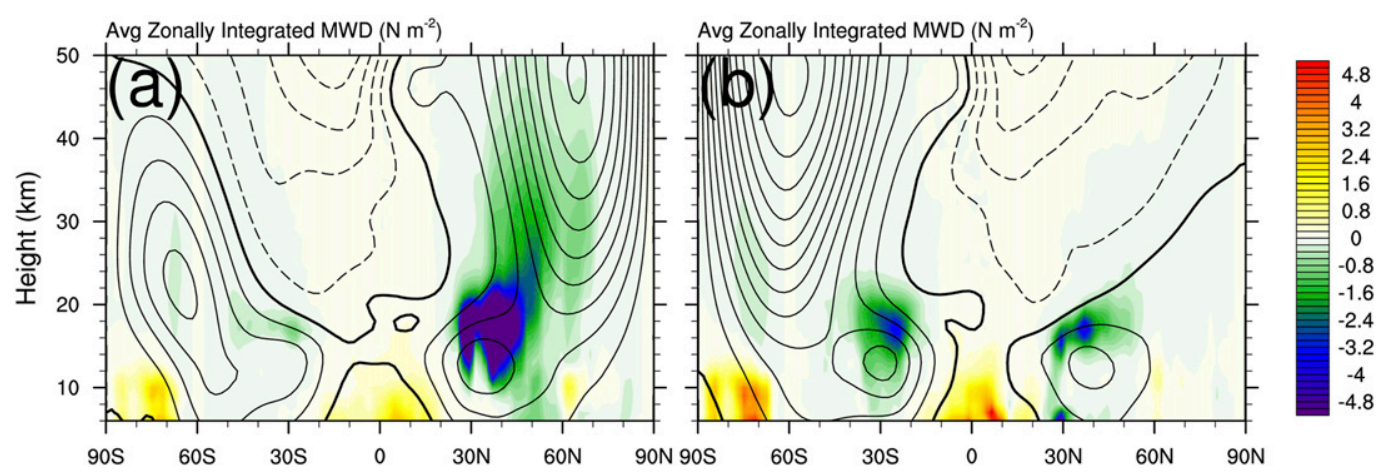

FIG. 3. Zonally integrated MWD $\left(\mathrm{N} \mathrm{m}^{-2}\right)$ averaged over the 90 days of integration and over all 20 branches with MWD (shading). Zonal, 90-day, and branch averaged zonal winds $\left(\mathrm{m} \mathrm{s}^{-1}\right)$ within the MWD branches are also shown (contoured every $10 \mathrm{~m} \mathrm{~s}^{-1}$, with solid positive contours and dashed negative contours). Results from (a) northern and (b) southern winter branches are shown.

where $\boldsymbol{\omega}_{a}$ is the absolute vorticity vector, $\chi(\theta)=\theta^{(1-\alpha)} /$ $\left[(1-\alpha) \theta_{0}^{-\alpha}\right]$ is a function of potential temperature $\theta$, $\theta_{0}=350 \mathrm{~K}, \alpha=1+c_{p} / R_{d} \approx 9 / 2, c_{p}$ is the specific heat capacity at constant pressure, $R_{d}$ the gas constant for dry air, $\rho$ is the density, and EPV classical Ertel PV. This form of PV is described by Lait (1994), which removes the exponential vertical increase in EPV, allowing more straightforward comparison of $\mathrm{PV}$ at different altitudes. The conservation equation of this $\mathrm{PV}$ is

$$
\frac{D \mathrm{PV}}{D t}=\frac{\boldsymbol{\omega}_{a} \cdot \nabla \dot{\chi}}{\rho}+\frac{\nabla \chi}{\rho} \cdot(\nabla \times \mathbf{F})
$$

where $D / D t$ is the material derivative, $\dot{\chi}=D \chi / D t$ is the diabatic tendency of $\chi$, and $\mathbf{F}$ represents viscous and/or other nonconservative body forces per unit mass. While radiative tendencies do influence $\mathrm{PV}$ evolution in the stratosphere on large time and space scales, the focus here is on the last term, through which MWD parameterizations create positive (negative) PV to the left (right) of a horizontal MWD vector. Quantitatively, MWD is defined here by

$$
\text { MWD }=-\frac{\partial \mathbf{M F}}{\partial z},
$$

where $\mathbf{M F}=\bar{\rho}\left(\overline{u^{\prime} w^{\prime}}, \overline{v^{\prime} w^{\prime}}\right)$. The nonconservative body force in Eq. (4) is related to MWD by $\mathbf{F}=\mathbf{M W D} / \bar{\rho}$, hereafter referred to as MWD tendency having units of deceleration.

\section{PV evolution in WACCM}

Zonally integrated, winter (i.e., three-month) averaged, ensemble-mean MWD in the MWD runs is shown for both $\mathrm{SH}$ and $\mathrm{NH}$ winter in Fig. 3. According to the parameterization, the strongest MW breakdown and corresponding drag force is exerted in the negative shear region above the subtropical jet in both hemispheres, where the environment climatologically approaches a critical level for zero phase speed MWs. GW-resolving limited area and global simulations of actual MW events suggest that strong MW breaking and drag deposition does occur in this region (Kruse et al. 2016; Niekerk et al. 2018), termed the MW valve layer by Kruse et al. (2016). Because of this peak in MWD, the primary level of interest here is $z=18 \mathrm{~km}$.

The evolution of $z=18 \mathrm{~km}$ PV differences over the first 15 days of the first $\mathrm{NH}$ branch $\left(\Delta \mathrm{PV} \mathrm{V}_{1}\right)$ is shown in Fig. 4. At 2.5 days after the branch, regional PV banners are evident, being advected downstream of contoured regions of PV generation $\left[\rho^{-1} \nabla \chi \cdot(\nabla \times \mathbf{F})\right]$ over the Himalayas and the Rockies. Resolved variability eventually distorts these regional PV banners. Gradually, $\Delta \mathrm{PV}_{1}$ due to different variability in the MWD and NoMWD runs appear, and by 15 days after the branch date, it is difficult to distinguish PV differences due to the introduction of parameterized MWD or due to solution divergence.

To see the evolution of MWD impacts further in time, PV differences are averaged over all 20 branches (i.e., $\widehat{\triangle P V}$ is computed). Again, this is equivalent to computing ensemble-mean PV fields in the MWD and NoMWD runs and then differencing. Within, for example, the NoMWD winters, different flow patterns are certainly present at a particular time of interest. The assumption is that the variability present at a particular time is random, averaging out over the 20 branches. In the MWD winters, variability is again averaged out, but systematic differences relative to the NoMWD winters remain due to the introduction of the MWD physics. Differencing the ensemble-mean MWD and NoMWD 


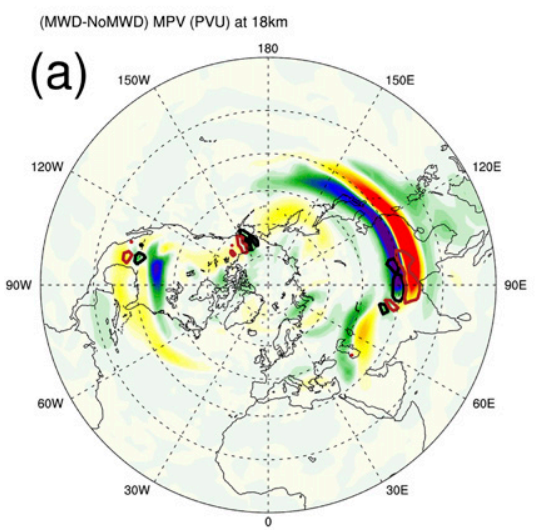

10.00 Days Since Branch at 20001201

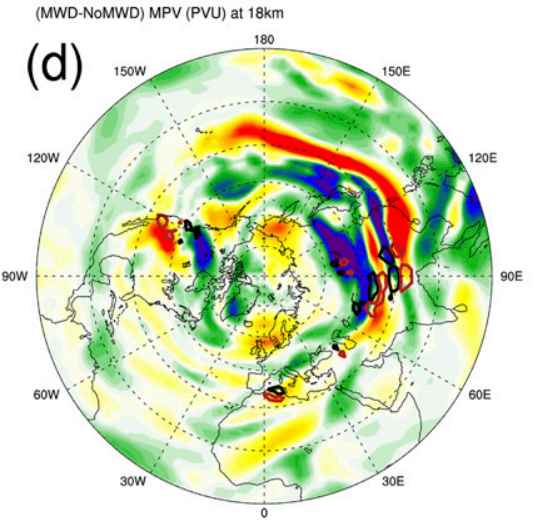

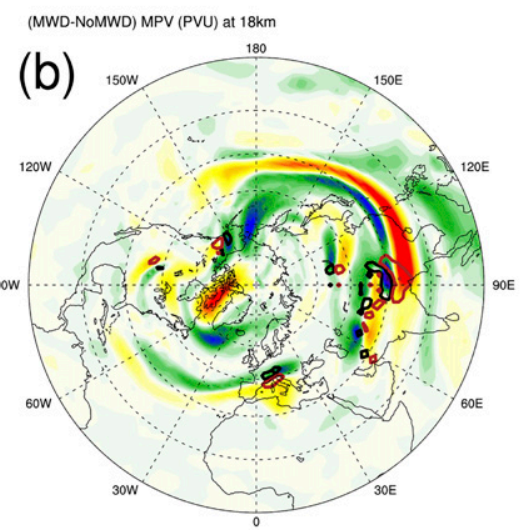

12.50 Days Since Branch at 20001201
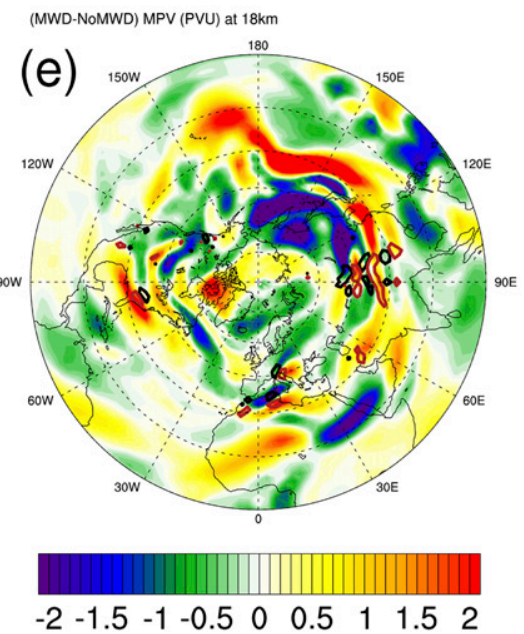



15.00 Days Since Branch at 20001201

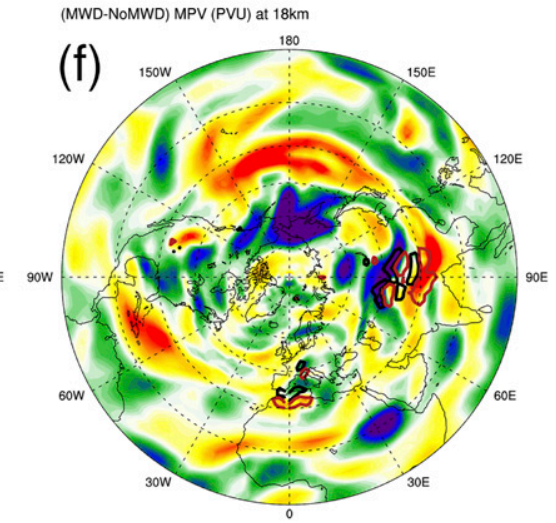

FIG. 4. MWD - NoMWD differenced PV fields at $z=18 \mathrm{~km}$ over the first 15 days in the first $\mathrm{NH}$ winter branch (i.e., $\Delta \mathrm{PV} \mathrm{V}_{1}$ ). $\mathrm{PV}$ generation by MWD [last term in Eq. (4)] is shown in contours $\left[2.5\right.$ and -2.5 (PVU day $\left.{ }^{-1}\right)\left(1 \mathrm{PVU}=10^{-6} \mathrm{~K} \mathrm{~kg}^{-1} \mathrm{~m}^{2} \mathrm{~s}^{-1}\right)$ in dark red and black contours, respectively).

runs then give the average or climatological influence of the MWD parameterization on PV.

The winter-long evolution of $\widetilde{\Delta \mathrm{PV}}$ created by the MWD parameterization and the corresponding $\widehat{\Delta U}$ are shown for NH (SH) winter in Fig. 5 (Fig. 6). Here, these ensemble-mean quantities are smoothed temporally with a 10-day moving average smoother.

In the $\mathrm{NH}$, a number of mountain ranges play a role in generating PV (best seen in Fig. 4). However, two mountain ranges stand out: the Himalayas, generating the most PV by far, and the Rockies (Fig. 5). Focusing first on the equatorward, cyclonic PV, these ranges generate cyclonic PV banners that are advected downstream within the first 10 days of the branch date, as noted before. By 20 days after the branch date, the cyclonic PV banners have been advected $\approx 90^{\circ}$ around the $\mathrm{NH}$, being apparently diffused along the way. By 35 days after the branch date, these banners have reached mountain ranges downstream, which reinforce these banners. The MWD-generated cyclonic PV advects and accumulates zonally into a zonal-mean or global feature. From this point onward, the cyclonic PV banner is continually generated, advected, diffused, and reinforced over downstream mountain ranges.

The anticyclonic, poleward PV is similarly advected downstream and around the pole. However, by 20 days after the branch time (Fig. 5b), the ensemble-mean, temporally smoothed anticyclonic PV banner is clearly more diffuse than the equatorward, cyclonic PV banner. Still, the anticyclonic PV is advected around the pole, diffused, but reinforced over downstream mountain ranges, eventually accumulating into a zonal-mean feature as well by 35 days after the branch time. Interestingly, beyond 35 days, the anticyclonic PV banner further diffuses over the pole, generating an anticyclonic PV polar cap. 
Avg over 0 to 10 days since Branch

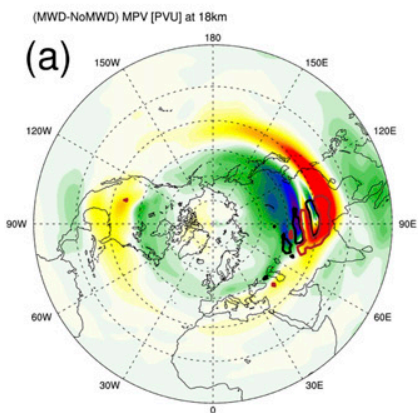

Avg over 45 to 55 days since Branch
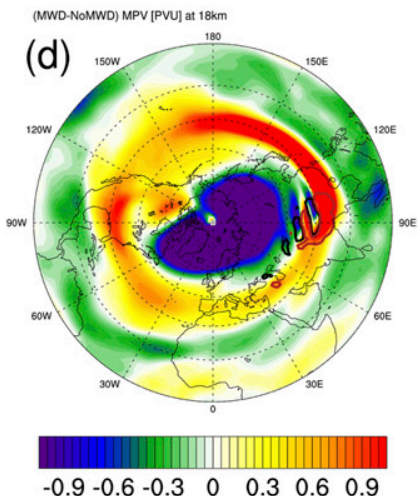
Avg over 0 to 10 days since Branch



Avg over 45 to 55 days since Branch

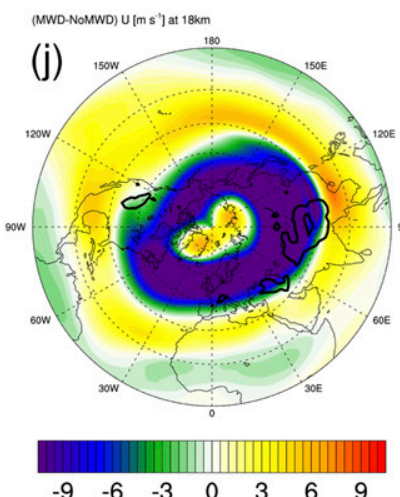

Avg over 15 to 25 days since Branch

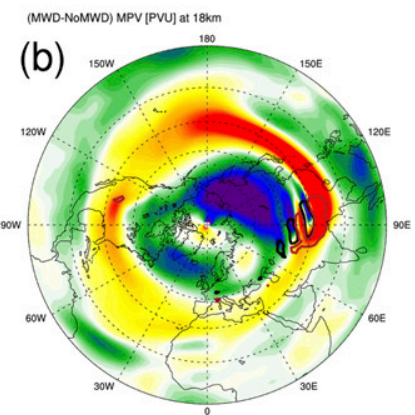

Avg over 60 to 70 days since Branch

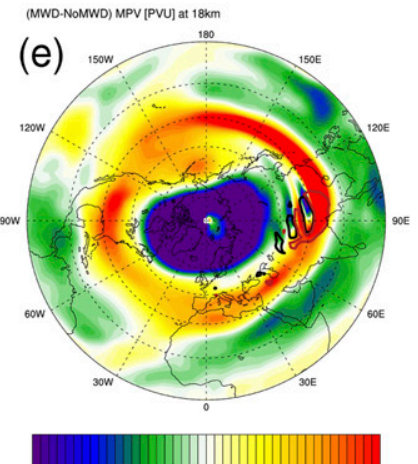

$\begin{array}{lllllll}-0.9 & -0.6 & -0.3 & 0 & 0.3 & 0.6 & 0.9\end{array}$ Avg over 15 to 25 days since Branch

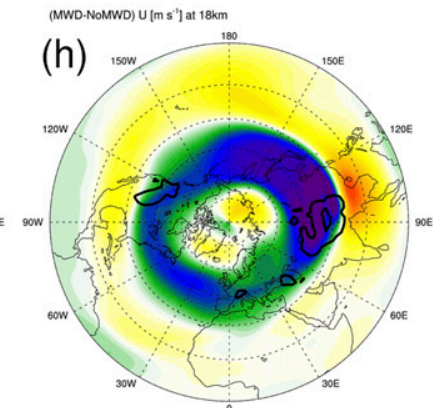

Avg over 60 to 70 days since Branch

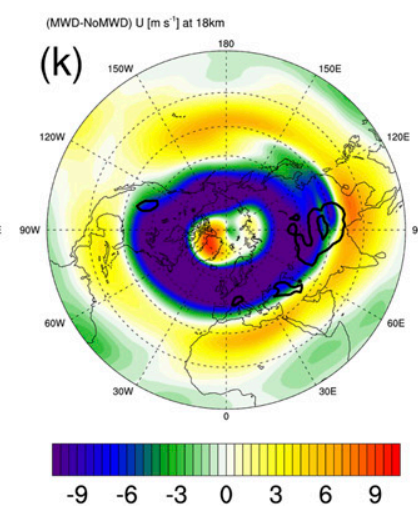

Avg over 30 to 40 days since Branch

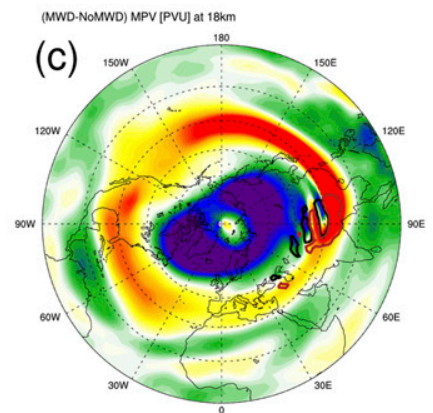

Avg over 75 to 85 days since Branch
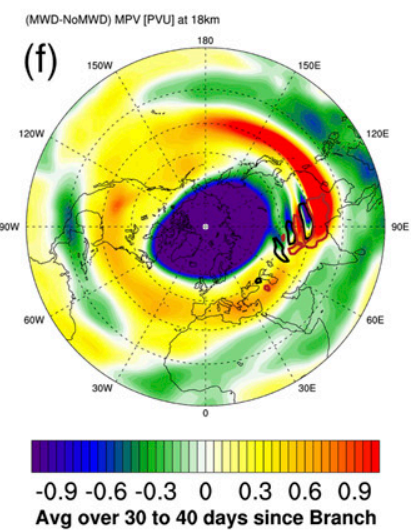

Avg over 30 to 40 days since Branch

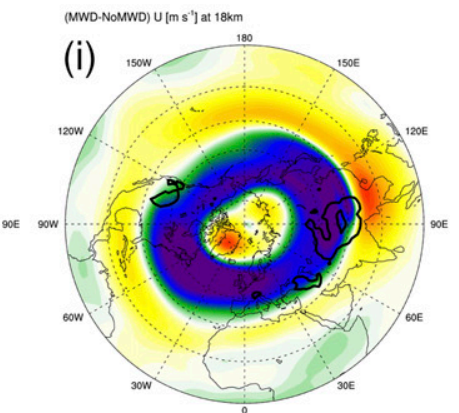

Avg over 75 to 85 days since Branch

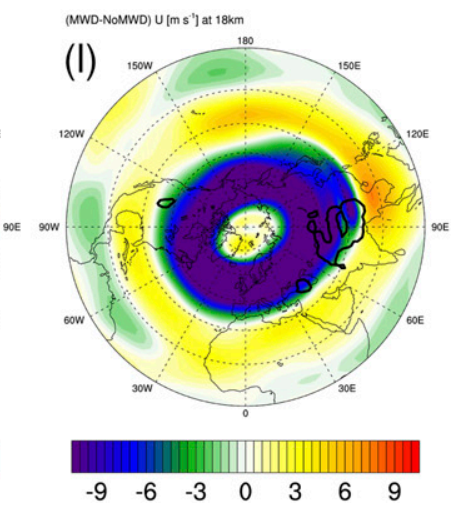

FIG. 5. (top two rows) $\widetilde{\Delta P V}$ and (bottom two rows) $\widetilde{\Delta U}$ evolution at days 5, 20, 35, 50, 65, and 80 days since branch at $z=18 \mathrm{~km}$ for the NH winter. Fields were differenced, averaged over all 20 branches, and further averaged over 10 days. The -1 (1) $\left(\mathrm{PVU}_{\text {day }}{ }^{-1}\right) \mathrm{PV}$ tendency contours are shown in black (dark red) in the top two rows. The $-5\left(\mathrm{~m} \mathrm{~s}^{-1} \mathrm{day}^{-1}\right)$ MWD tendency contour is shown in black in the bottom two rows. 


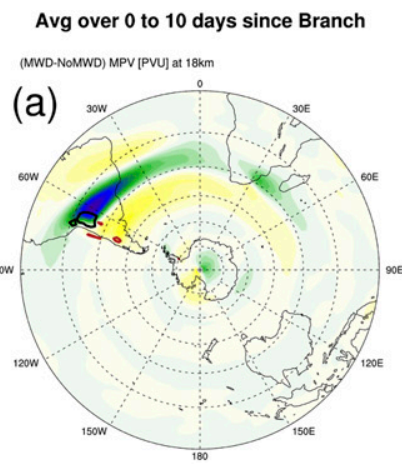

Avg over $\mathbf{4 5}$ to $\mathbf{5 5}$ days since Branch


Avg over $\mathbf{4 5}$ to 55 days since Branch
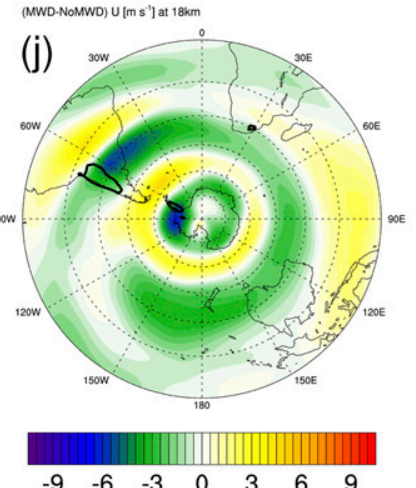

Avg over 15 to 25 days since Branch

(MWD-NoMWD) MPV [PVU] at $18 \mathrm{~km}$

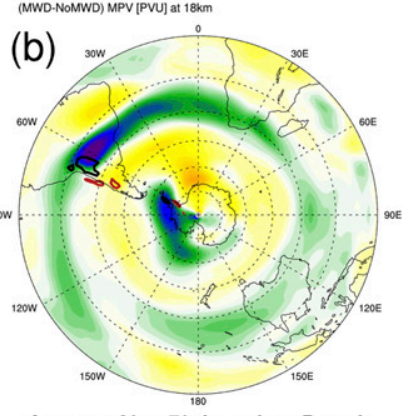

Avg over 60 to 70 days since Branch

(e)

(2)


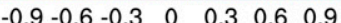
Avg over 15 to 25 days since Branch

(MWD-NoMWD) U [m s'] at $18 \mathrm{~km}$

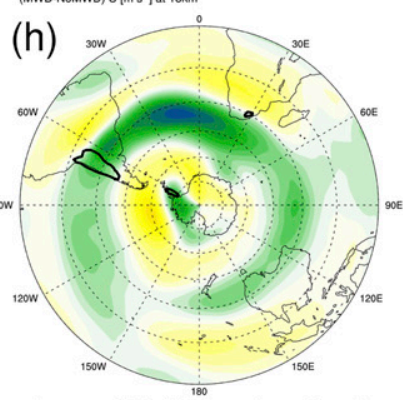

Avg over 60 to 70 days since Branch

(MWD-NoMWD) U [m s'] at 18km

(k)
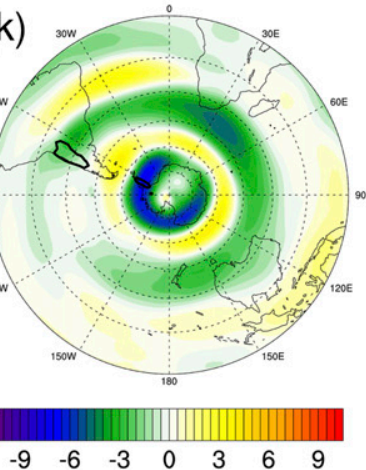

Avg over 30 to 40 days since Branch

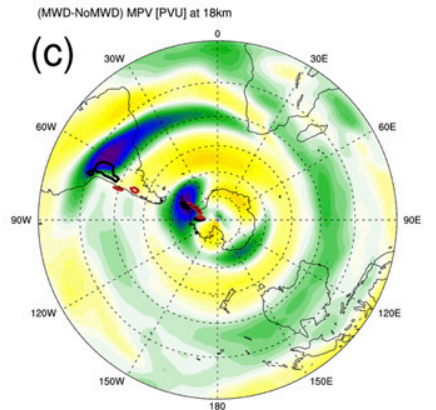

Avg over 75 to 85 days since Branch
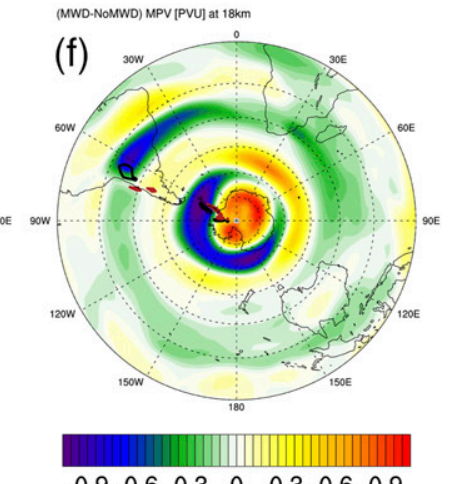

$\begin{array}{lllllll}-0.9 & -0.6 & -0.3 & 0 & 0.3 & 0.6 & 0.9\end{array}$ Avg over 30 to 40 days since Branch

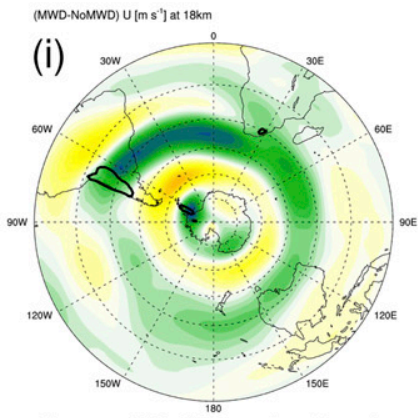

Avg over 75 to 85 days since Branch (MWD-NoMWD) U [m s'] at 18km
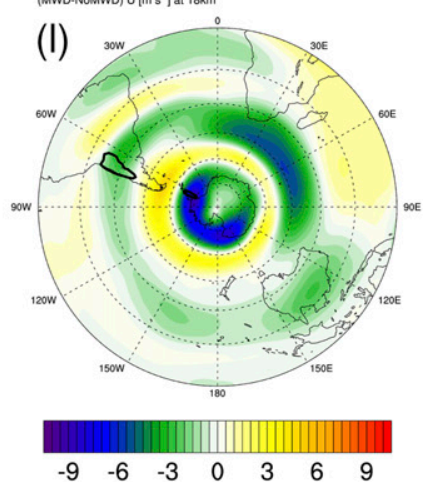

FIG. 6. As in Fig. 5, but for over the SH winter. 
The zonal wind decelerations that accompany the evolution of these PV banners are substantial, exceeding $10 \mathrm{~m} \mathrm{~s}^{-1}$, and also accumulating zonally into a global feature. After day 50, the magnitude of at least the cyclonic PV banner appears reduced. This reduction in PV generation is possibly due to the fact that wind speed reductions associated with PV advected from upstream mountain ranges cause more wave breaking below and less MWD at $z=18 \mathrm{~km}$. The parameterization formulation results in MWD being proportional to $U^{3}$ (Lindzen 1981; McFarlane 1987), suggesting the parameterized MWD should be quite sensitive to the decelerations seen in Figs. 5g-1. Seasonal variation in MWD at this level might impact PV generation and banner magnitude at these later times as well.

The $\mathrm{SH}$ is different from the NH in two key ways. The primary generators of MWD and PV at this level (Andes, Antarctic Peninsula) have a very different geographic configuration (similar longitude, different latitude) and are weaker than the NH (Fig. 6). Still, both generate positive and negative PV. Focusing on the Andean PV banners, the equatorward, cyclonic PV again appears less diffused than the poleward, anticyclonic PV banner. Both are advected eastward, and are somewhat reinforced over the South African mountains. By day 50, the Andean PV banners have accumulated zonally into global, though weak, features with zonal wind decelerations of -3 to $-6 \mathrm{~m} \mathrm{~s}^{-1}$.

While the mountains on the Antarctic Peninsula certainly generate MWD at this level, the fate of PV generated there is less clear. While zonally symmetric PV and zonal wind differences develop with latitudes corresponding to this region of MWD, these differences do not appear directly tied to regions of generation over the Antarctic Peninsula. These PV features might instead be indirect effects of turning on MWD, due to a displacement of the polar vortex northward along $135^{\circ} \mathrm{W}$ (note negative $\Delta \mathrm{PV}$ upstream of the Antarctic Peninsula in Fig. 6).

The primary result of this section, and paper, is that, on average, PV generated by MWD is advected downstream creating continent-scale PV banners. These banners are apparently diffused, but this diffusion does not prevent the zonal accumulation of these continent-scale PV banners into global features. Numerous questions remain, however. What is the cause of this diffusion? Why do the PV banners not diffuse away? Why are the poleward and equatorward banners diffused differently? Can simple horizontal, 2D diffusion explain the diffusion apparent in these WACCM results? These questions are entertained in the following section using a simple, idealized 2D diffusion model.

\section{Horizontal diffusion of "passive PV"}

The WACCM results of the previous section reveal the regional to global evolution of the influences of MWD. Here, the hypothesis that the evolution can be explained by a source, advection, and horizontal diffusion process is tested using an imperfect, but understandable, 2D advection/diffusion model.

\section{a. 2D diffusion model}

Here, PV is treated as a passive scalar that is generated, horizontally advected, and horizontally diffused via the following governing equation:

$$
\frac{\partial \mathrm{PV}}{\partial t}=-\mathbf{V} \cdot \nabla \mathrm{PV}+\nabla \cdot(\kappa \nabla \mathrm{PV})+\mathrm{PV} .
$$

This equation is discretized on a $30000 \mathrm{~km}$ by $30000 \mathrm{~km}$ doubly periodic domain with a resolution of $100 \mathrm{~km}$, about twice the resolution of WACCM. Fourth-order finite differences were used to compute spatial derivatives. Time integration was performed using a fourthorder Runge-Kutta scheme.

The inner $\pm 10000 \mathrm{~km}$ by $\pm 10000 \mathrm{~km}$ section of the domain is considered. This choice was made because the distance between the equator and the pole is $\approx 10000 \mathrm{~km}$. This region has an area and circumferences comparable, though not equal, to those on a hemisphere of the Earth. For example, the area of this region is a somewhat smaller $\left(10^{8} \mathrm{~km}^{2}\right)$ than the area of a hemisphere $\left(2.55 \times 10^{8} \mathrm{~km}^{2}\right)$. The circumference of a circle with some radius in the $2 \mathrm{D}$ domain is somewhat larger than the circumference of a latitude circle with a distance from the pole equal to this radius. The ratio of these two circumferences is $C_{2 \mathrm{D}} / C_{\mathrm{Sph}}=r /\left[r_{e} \sin \left(r / r_{e}\right)\right]$. The distance traveled by a parcel advected around the $2 \mathrm{D}$ domain at a distance of $10000 \mathrm{~km}$ from the center is a factor of 1.57 longer than a parcel advected around the equator.

\section{1) Passive PV Generation}

Passive PV generation is represented by PV. The PV field is calculated from an azimuthal MWD tendency field, $\mathbf{F}=\left(0, F_{y}\right)$, where

$$
F_{y}=\left\{\begin{array}{cc}
\frac{F_{\max }}{2}\left[1+\cos \left(2 \pi \frac{r_{1}}{L_{\mathrm{PV}}}\right)\right], & r_{1} \leq \frac{L_{\mathrm{pv}}}{2} \\
0, & r_{1}>\frac{L_{\mathrm{pv}}}{2}
\end{array},\right.
$$

$r_{1}=\sqrt{x_{1}^{2}+y_{1}^{2}},\left(x_{1}, y_{1}\right)=\left(x-x_{0}, y-y_{0}\right),\left(x_{0}, y_{0}\right)$ is the center of the region of MWD tendency, $L_{\mathrm{PV}}$ is the diameter of this region, and $F_{\max }$ is the maximum MWD tendency. PV generation is given by 



FIG. 7. (top) A comparison between NH winter WACCM fields at $z=18 \mathrm{~km}$ and (bottom) corresponding fields in the 2D diffusion model. WACCM fields are from the MWD runs and were averaged over the winter (i.e., three months) and all 20 branches. WACCM (a) PV generation (PVU day ${ }^{-1}$ ) and (b) zonal winds ( $\mathrm{m} \mathrm{s}^{-1}$ ) are shown in color shading. Zonal winds are contoured in and (b) as well. (c) Passive PV generation (PVU day ${ }^{-1}$ ) and (d) azimuthal wind $\left(\mathrm{m} \mathrm{s}^{-1}\right)$ in the $2 \mathrm{D}$ diffusion model are also shown.

$\mathrm{PV}=\frac{\nabla \chi}{\rho} \cdot(\nabla \times \mathbf{F})=\frac{1}{\rho} \frac{\partial \chi}{\partial z} \frac{\partial F_{y}}{\partial x}=\frac{1}{\rho}\left(\frac{\theta}{\theta_{0}}\right)^{-\alpha} \frac{\theta}{g} N^{2} \frac{\partial F_{y}}{\partial x}$,

where $N=0.02 \mathrm{~s}^{-1}, \rho=0.12 \mathrm{~kg} \mathrm{~m}^{-3}, \theta=454 \mathrm{~K}$, and $\theta_{0}=350 \mathrm{~K}$.

To construct a PV field similar to NH tendencies in WACCM, the following values were assigned to undefined constants above: $F_{\max }=20 \mathrm{~m} \mathrm{~s}^{-1}$ day $^{-1}$, $\left(x_{0}, y_{0}\right)=(-5500,0) \mathrm{km}, L_{\mathrm{PV}}=3000 \mathrm{~km}$. This distance from the origin is similar to the distance between the Himalayas (or Rockies) and the North Pole. The MWD tendencies in the $\mathrm{NH}$ case are given by $F_{y_{\mathrm{NH}}}=F_{y}-2 F_{y}^{\mathrm{T}}\left[(.)^{\mathrm{T}}\right.$ the transpose], which gives two PV sources $180^{\circ}$ apart with one twice as strong as the other representing the Rockies and Himalayas. In the $\mathrm{SH}$, two sources are again specified, but both at negative $x:\left(x_{0_{1}}, y_{0_{1}}\right)=(-5500,0) \mathrm{km},\left(x_{0_{2}}, y_{0_{2}}\right)=(-2000,0) \mathrm{km}$, $F_{\max _{1}}=F_{\max _{2}}=-20 \mathrm{~m} \mathrm{~s}^{-1}$ day $^{-1}, L_{\mathrm{PV}_{1}}=3000 \mathrm{~km}$, and $L_{\mathrm{PV}_{2}}=1500 \mathrm{~km}$. The SH MWD tendencies are then $F_{y_{\mathrm{SH}}}=F_{y_{1}}+F_{y_{2}}$. Passive PV tendencies were then computed via Eq. (8). The $\mathrm{NH}(\mathrm{SH}) \mathrm{PV}$ tendencies in
WACCM are compared with the passive PV representation in Figs. 7a,c and 8a,c.

\section{2) VORTEX SPECIFICATION}

Vortices similar to those seen at $z=18 \mathrm{~km}$ in WACCM are specified to advect the PV created by the PV fields. These vortices are not coupled to PV in any way; hence the PV modeled by Eq. (8) is called passive PV. The magnitude of the vortex winds are given by

$$
\mathrm{WS}=\left\{\begin{array}{cc}
\frac{\mathrm{WS}_{\mathrm{max}}\left[1-\cos \left(2 \pi \frac{r}{L_{\mathrm{wS}}}\right)\right],}{} & r \leq L_{\mathrm{wS}}, \\
0, & r>L_{\mathrm{ws}}
\end{array}\right.
$$

where $r$ is the distance from the origin (i.e., the pole), $\mathrm{WS}_{\max }=30(50) \mathrm{m} \mathrm{s}^{-1}$, and $L_{\mathrm{WS}}=10000(9000) \mathrm{km}$ in the NH (SH) cases. The vortex spins counterclockwise (clockwise) in the NH (SH). The NH (SH) WACCM and 2D model vortices compared Figs. 7b,d and 8b,d. 

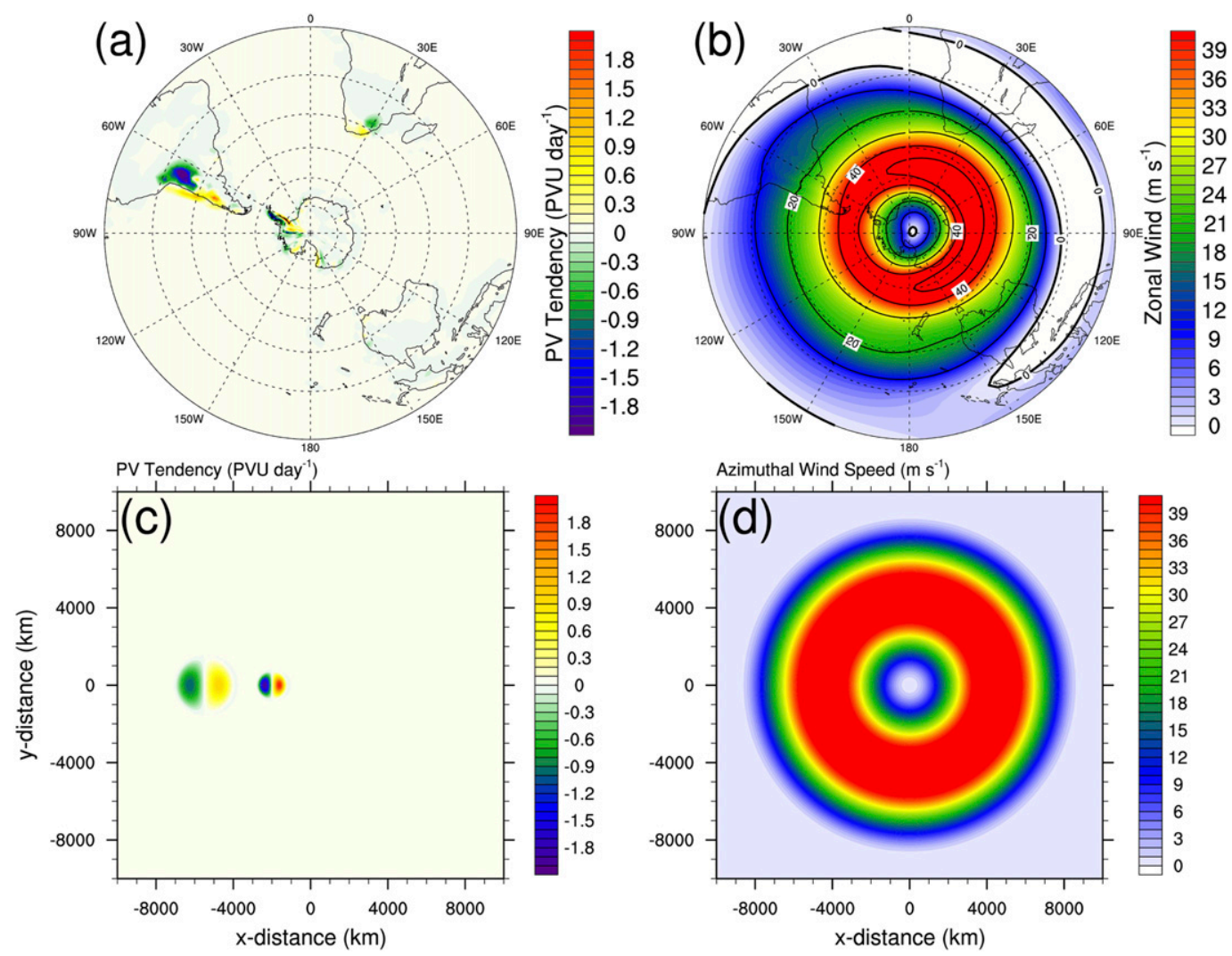

FIG. 8. As in Fig. 7, but for the SH.

The vortex at this level is considerably stronger and somewhat more compact in the SH.

\section{3) PASsive PV DIFFUSION}

The final element of the simple model yet to be defined is the diffusivity. Here, the speculation is that PV diffusion, both parameterized and numerical, is enhanced by resolved variability (e.g., Rossby waves). This resolved variability acts to stir the MWD-generated PV banners, increasing small-scale gradients along sheared and stretched contours, thereby increasing the diffusive or mixing ability of the flow (Nakamura 1996). Haynes and Shuckburgh (2000) investigated how stratospheric resolved variability enhances "microscale" diffusivity of passive scalars (i.e., turbulent or numerical diffusivities in this context) in the meridional direction in a zonalmean sense, quantified by an "effective diffusivity." They showed that reanalyzed flow fields on an isentrope can significantly enhance meridional diffusion of passive scalars, with effective diffusivities locally an order of magnitude larger than the prescribed microscale diffusivity. In short, an analogy is drawn between the apparent horizontal diffusion of PV in WACCM and with concepts of 3D turbulence, where the largest eddies produce the largest turbulent fluxes, while subsequent shearing, stretching, and nonlinear dynamics generating smaller and smaller scales of motion allow molecular diffusivity to act. In the WACCM simulations presented here, it is speculated that the $2 \mathrm{D}$ resolved variability (e.g., Rossby waves) distort the MWDgenerated PV banners until parameterized and numerical horizontal diffusion can act.

A constant diffusivity of $\kappa=10^{6} \mathrm{~m}^{-2} \mathrm{~s}^{-1}$ is used to represent the effects of the resolved, largely $2 \mathrm{D}$ turbulence in WACCM in both hemispheres. This value is arrived at by assuming PV is diffused by eddies with characteristic length and velocity scales of $100 \mathrm{~km}$ and $10 \mathrm{~m} \mathrm{~s}^{-1}$, respectively, and is consistent with, though somewhat smaller than, the effective diffusivities presented by Haynes and Shuckburgh (2000). While a radially varying (effective) diffusivity might be used to represent latitudes with increased resolved variability and horizontal mixing in the stratosphere (e.g., the surf zone), a constant diffusivity was chosen so that the complexity of this already crude model would not be unnecessarily increased. That said, using a diffusivity varying radially similar to how effective diffusivities presented by Haynes and Shuckburgh (2000) vary with 

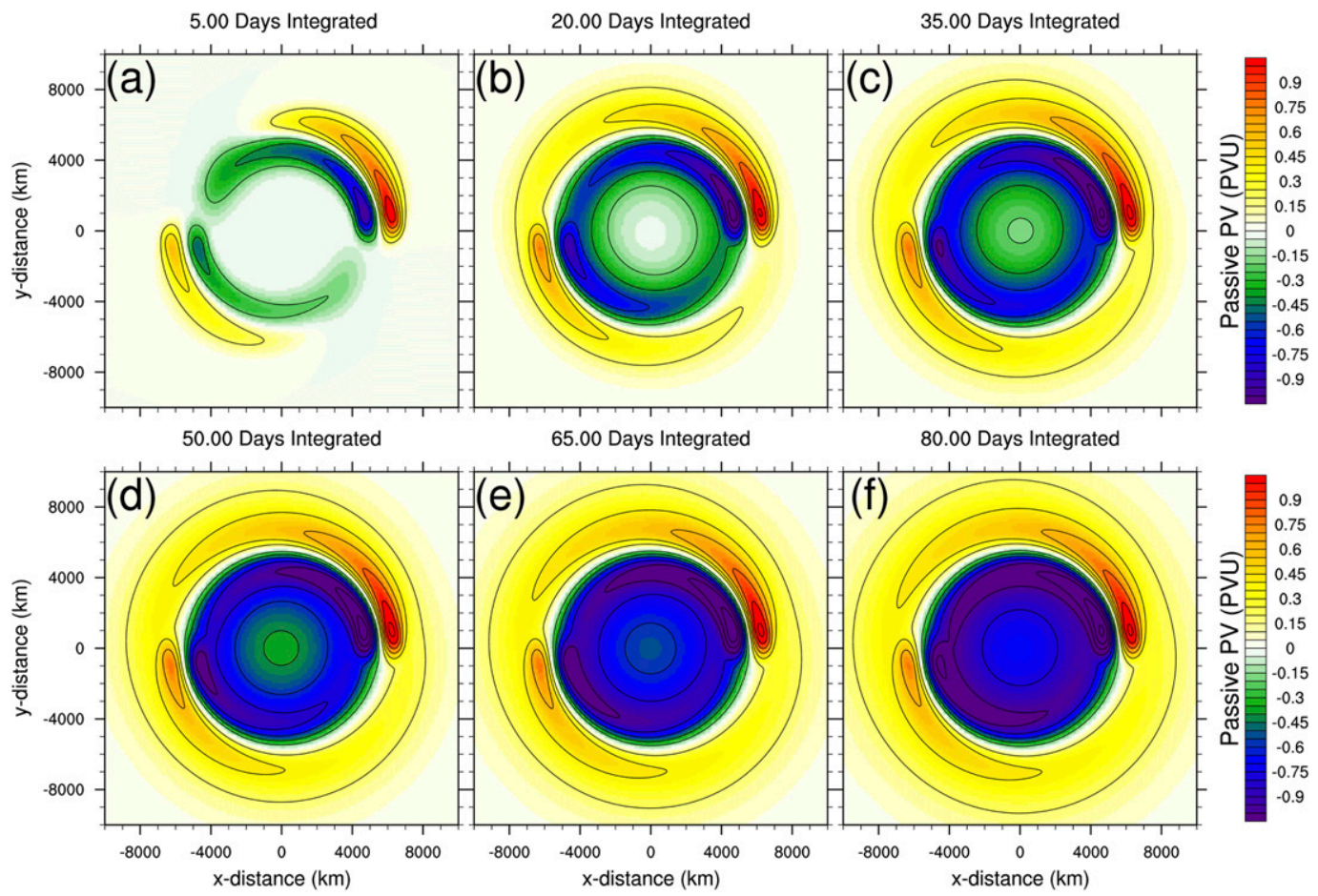

FIG. 9. Passive PV (PVU) evolution at days 5, 20, 35, 50, 65, and 80 days integrated by the 2D diffusion model in the NH configuration. Passive PV is color shaded and contoured every 0.2 PVU.

latitude did not significantly change solutions of this $2 \mathrm{D}$ model (not shown).

\section{4) 2D MODEL LIMITATIONS}

Using such a simple model to understand the evolution of PV in WACCM does have shortcomings. For example, the 2D Cartesian geometry of the domain is similar to, but not the same as that of a hemisphere of a spherical shell. The PV being generated and advected is passive, not influencing the winds that advect it. This issue would not be significant if the balanced flow associated with the MWD-generated PV were small. However, the decelerations associated with MWD-generated PV can be comparable in magnitude to the background winds advecting it (e.g., cf. Figs. 5g-1 and 3a), suggesting that the balanced flow associated with this PV may be important in its evolution. Additionally, use of a constant eddy diffusivity to represent the diffusive influences of resolved variability is quite crude. However, as will be shown, this model reproduces a number of salient features in the evolution of the dynamic PV in WACCM quite well, allowing the evolution of MWD influences to be better understood.

\section{b. Passive PV evolution}

The winter-long evolution of $\mathrm{NH}$ passive $\mathrm{PV}$ is shown in Fig. 9. Overall, there is qualitative agreement with the evolution of dynamic PV in WACCM (cf. Fig. 5). PV banners are advected downstream of both PV sources, are diffused, but then reinforced once the banners reach the other region of PV generation downstream.

Why do the banners not diffuse away? To answer this question, the ratio of the advective time scale to the diffusion time scale is estimated, here referred to as the axisymmetrization ratio:

$$
R_{\mathrm{axi}}=\frac{\tau_{\mathrm{adv}}}{\tau_{\kappa}}=\frac{C(r)}{U(r)} \frac{\kappa}{\left(L_{\mathrm{PV}} / 2\right)^{2}} .
$$

This is an inverse Péclet number. Here, $C(r)$ is the distance between PV sources. The distance between the maximum and minimum passive PV generation is taken to be the relevant length scale over which diffusion is considered here. When $R_{\mathrm{axi}}<1$, the advection time scale is smaller than the diffusion time scale, and passive PV can axisymmetrize, generating annular features. When $R_{\text {axi }}>1$, diffusion dominates, and diffusive fluxes between the two banners annihilate the banners, preventing the development of annular features. For the $\mathrm{NH}(\mathrm{SH})$ passive PV banners, $R_{\mathrm{axi}} \approx 0.76(1.54)$, using $r=5500 \mathrm{~km}, U(r)=10 \mathrm{~m} \mathrm{~s}^{-1}, \kappa=10^{6} \mathrm{~m}^{2} \mathrm{~s}^{-1}$ and $L_{\mathrm{PV}}=$ $3000 \mathrm{~km}$. For the NH, half the circumference was used as the two sources are $180^{\circ}$ apart. These axisymmetrization ratios suggest annular features should develop in the $\mathrm{NH}$ 


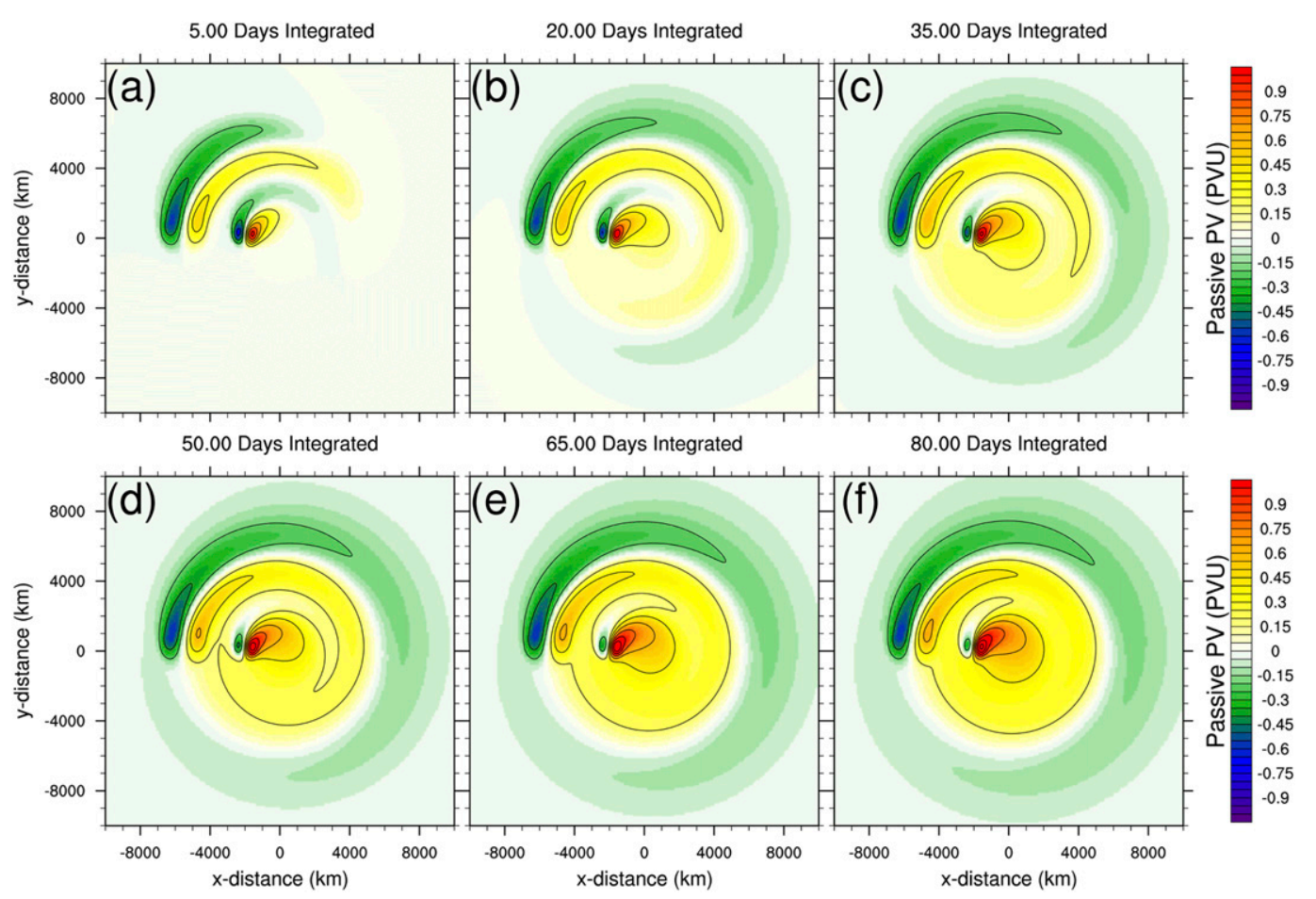

FIG. 10. As in Fig. 9, but with the 2D diffusion model in the SH configuration.

case, while passive PV banners in the $\mathrm{SH}$ case should be significantly influenced by diffusion, consistent with Figs. 9 and 10.

Another feature present in both the 2D and WACCM solutions is the apparent asymmetric diffusion of the cyclonic and anticyclonic banners. The equatorward (i.e., further from the domain origin here), cyclonic passive PV banners are advected, diffused, and reinforced, but generally accumulate and stay within an annulus (Fig. 9), similar to the WACCM results (Figs. 5a-f). The evolution of the anticyclonic, poleward passive $\mathrm{PV}$ in the 2D model is also similar to the evolution in WACCM. The poleward passive PV also quickly accumulates into an annular feature but then, interestingly, diffuses over the pole into an anticyclonic PV polar cap.

In short, the fast poleward diffusion results from anticyclonic fluxes into annuli/regions of smaller area near the pole, resulting in larger tendencies there. For example, the PV tendency within an infinitesimally thin, axisymmetric annulus is given by

$$
\frac{\partial \mathrm{PV}}{\partial t}=\kappa\left(\frac{\partial^{2} \mathrm{PV}}{\partial r^{2}}+\frac{1}{r} \frac{\partial \mathrm{PV}}{\partial r}\right)
$$

where $\kappa$ was taken to be constant. Consider an axisymmetric negative passive $\mathrm{PV}$ banner around the pole: $\mathrm{PV}(r)=-\left\{(A / 2)\left[1-\cos \left(k_{r} r\right)\right]\right\}$, where $A$ is the amplitude of the banner, $k_{r}=2 \pi / L_{r}$ is the radial wavenumber of the banner with a wavelength of $L_{r}$. On the edges of the banner (i.e., distances $L_{r} / 4<\left|r_{c}\right|<L_{r} / 2$, with $r_{c}=$ $r-L_{r} / 2$ the distance from the point of minimum PV), the curvature term in Eq. (11) is negative and symmetric about the center of the banner. Considering a $2 \mathrm{D}$ banner in Cartesian coordinates [i.e., $\mathrm{PV}(x)$ rather than $\mathrm{PV}(r)$ ], diffusion would produce equivalent, negative PV tendencies on the edges of the banner. But in the polar geometry considered here, the gradient term enhances (reduces) tendencies on the poleward (outward) side of the banner relative to the 2D Cartesian banner. The magnitude of the gradients is the same (though negative poleward, positive outward), but the $1 / r$ factor in the gradient term significantly enhances the importance of this term on the poleward side, resulting much larger negative tendencies and faster apparent poleward diffusion of this PV.

In the SH, Andean PV banners nearly accumulate into annular features, but as noted above $\left(R_{\mathrm{axi}}=1.54\right)$, the banners are not reinforced halfway around the domain and are nearly diffused away by the time they circumnavigate the pole. The anticyclonic, poleward PV banner again diffuses more quickly poleward. For the PV generation representing the Antarctic Peninsula, the axisymmetrization ratio $\left(R_{\mathrm{axi}} \approx 1.12\right)$ suggests these banners would also be strongly diffused. The poleward diffusion of the anticyclonic Andean PV banner somewhat compensates the cyclonic PV banner from the 
Antarctic Peninsula after $\approx 30$ days. The poleward diffusion of the peninsula-generated anticyclonic PV banner also generates an anticyclonic PV polar cap, albeit smaller than in the $\mathrm{NH}$ case.

\section{Summary and discussion}

The experiment presented here has shown how regional MWD in the lower stratosphere induces a zonalmean or global response by tracking the climatological evolution of the PV it generates within WACCM. In short, PV is generated over major mountain ranges, is advected downstream creating continent-scale PV banners, is diffused, likely through mixing by resolved variability, but the banners survive this diffusion and accumulate into zonal-mean, global PV features. The equatorward, cyclonic PV banners are diffused less than the poleward, anticyclonic banners, accumulating into a single global cyclonic PV banner. Anticyclonic PV banners are quickly advected around the pole, generating a global anticyclonic PV banner. This banner is then continually reinforced by anticyclonic PV generation and diffused poleward. This poleward diffusion generates an anticyclonic PV polar cap. Zonal wind decelerations associated with the evolving PV are significant, in excess of $10 \mathrm{~m} \mathrm{~s}^{-1}$ in the $\mathrm{NH}$ and $3-6 \mathrm{~m} \mathrm{~s}^{-1}$ in the $\mathrm{SH}$.

A 2D source, advection, diffusion model for passive $\mathrm{PV}$ was constructed to better understand the long-term evolution of PV in WACCM. Despite the simplicity of this model, the behavior and evolution of passive PV had several similarities with the evolution of the dynamic PV in WACCM. With an order-of-magnitude estimate of diffusivity, the simple model-generated annular PV features that survive diffusion. The poleward PV banners diffused more quickly than the equatorward banners as well. This asymmetry is due to the polar/spherical geometry, where poleward diffusive fluxes of PV are into annuli/regions of smaller areas relative to the equatorward fluxes. Once the global anticyclonic PV banner is formed, it also diffuses over the pole, creating an anticyclonic PV polar cap.

It is worth noting that while the focus here was on the lower stratosphere, very significant influences of the MWD parameterization occur further aloft near the winter stratopause ( $z \approx 50 \mathrm{~km}$, Fig. 1). Despite weaker parameterized MWD there (Fig. 3), the much lower density allows for much larger decelerations and PV generation. The same processes governing the evolution of direct MWD impacts (i.e., evolution of MWDgenerated PV) in the lower stratosphere apply near the stratopause as well, though here, indirect MWD impacts (e.g., vortex displacements, altered resolved wave breaking) become important and complicate the view of the evolution of direct MWD impacts there (not shown). Future work is envisioned to document and understand both the indirect and direct impacts of MWD in the middle atmosphere.

Viewing the impacts of MWD in the PV framework not only revealed their regional to global evolution, the PV framework also allows MWD impacts to be readily incorporated into the existing zonal-mean picture with Rossby waves and other processes that influence the meridional structure of PV. For example, in the quasigeostrophic limit, the zonal-mean wave forcing (i.e., the Eliassen-Palm flux divergence) is equivalent to the meridional flux of quasigeostrophic PV. Within the winter stratosphere, both MWs and resolved waves flux PV downgradient (southward). How the MWD parameterization and resolved Rossby waves interact to modify the radiatively driven meridional PV field is an interesting question. More might be learned in a nonaxisymmetric frame as presented here.

Acknowledgments. This material is based upon work supported by the National Center for Atmospheric Research, which is a major facility sponsored by the National Science Foundation under Cooperative Agreement 1852977. This work was also supported early on by an NSF grant to Yale University (NSF-AGS1338655). High-performance computing was performed on the Cheyenne supercomputer (ark:/85065/d7wd3xhc) with support provided by NCAR's Computational and Information systems Laboratory, also sponsored by the National Science Foundation. Finally, I want to acknowledge the many useful discussions with Ronald B. Smith, Yun Chang, Julio Bacmeister, and Petr Šácha throughout this research.

\section{REFERENCES}

Chen, C., G. J. Hakim, and D. R. Durran, 2007: Transient mountain waves and their interaction with large scales. J. Atmos. Sci., 64, 2378-2400, https://doi.org/10.1175/JAS3972.1.

Haynes, P., and E. Shuckburgh, 2000: Effective diffusivity as a diagnostic of atmospheric transport: 1. Stratosphere. J. Geophys. Res., 105, 22 777-22 794, https://doi.org/10.1029/2000JD900093.

Kruse, C. G., R. B. Smith, and S. D. Eckermann, 2016: The midlatitude lower-stratospheric mountain wave "valve layer." J. Atmos. Sci., 73, 5081-5100, https://doi.org/10.1175/JAS-D-16-0173.1.

Lait, L. R., 1994: An alternative form for potential vorticity. J. Atmos. Sci., 51, 1754-1759, https://doi.org/10.1175/15200469(1994)051<1754:AAFFPV>2.0.CO;2.

Lindzen, R. S., 1981: Turbulence and stress owing to gravity wave and tidal breakdown. J. Geophys. Res., 86, 9707-9714, https:// doi.org/10.1029/JC086iC10p09707.

McFarlane, N. A., 1987: The effect of orographically excited gravity wave drag on the general circulation of the lower stratosphere and troposphere. J. Atmos. Sci., 44, 1775-1800, https://doi.org/ 10.1175/1520-0469(1987)044<1775:TEOOEG > 2.0.CO;2. 
Menchaca, M. Q., and D. R. Durran, 2018: The impact of mountain waves on an idealized baroclinically unstable large-scale flow. J. Atmos. Sci., 75, 3285-3302, https://doi.org/10.1175/JAS-D17-0396.1.

Miller, M. J., T. N. Palmer, and R. Swinbank, 1989: Parametrization and influence of subgridscale orography in general circulation and numerical weather prediction models. Meteor. Atmos. Phys., 40, 84-109, https://doi.org/10.1007/ BF01027469.

Nakamura, N., 1996: Two-dimensional mixing, edge formation, and permeability diagnosed in an area coordinate. J. Atmos. Sci., 53, 1524-1537, https://doi.org/10.1175/1520-0469(1996) 053<1524:TDMEFA $>2.0$. CO;2.

Neale, R. B., and Coauthors, 2010: Description of the NCAR Community Atmosphere Model (CAM5.0). NCAR Tech. Note NCAR/TN-486+STR, 268 pp., www.cesm.ucar.edu/models/ cesm1.1/cam/docs/description/cam5_desc.pdf.

Niekerk, A., I. Sandu, and S. B. Vosper, 2018: The circulation response to resolved versus parametrized orographic drag over complex mountain terrains. J. Adv. Model. Earth Syst., 10, 2527-2547, https://doi.org/10.1029/2018MS001417.
Palmer, T. N., G. J. Shutts, and R. Swinbank, 1986: Alleviation of a systematic westerly bias in general circulation and numerical weather prediction models through an orographic gravity wave drag parametrization. Quart. J. Roy. Meteor. Soc., 112 , 1001-1039, https://doi.org/10.1002/qj.49711247406.

Sandu, I., and Coauthors, 2019: Impacts of orography on largescale atmospheric circulation. npj Climate Atmos. Sci., 2, 10 , https://doi.org/10.1038/S41612-019-0065-9.

Schär, C., M. Sprenger, D. Lüthi, Q. Jiang, R. B. Smith, and R. Benoit, 2003: Structure and dynamics of an alpine potential-vorticity banner. Quart. J. Roy. Meteor. Soc., 129, 825-855, https:// doi.org/10.1256/qj.02.47.

Sigmond, M., and J. F. Scinocca, 2010: The influence of the basic state on the Northern Hemisphere circulation response to climate change. J. Climate, 23, 1434-1446, https://oi.org/10.1175/ 2009JCLI3167.1.

Smith, R. B., 1989: Hydrostatic airflow over mountains. Advances in Geophysics, Vol. 31, Academic Press, 1-41, https://doi.org/ 10.1016/S0065-2687(08)60052-7.

Teixeira, M. A. C., 2014: The physics of orographic gravity wave drag. Front. Phys., 2, 43, https://doi.org/10.3389/fphy.2014.00043. 